\title{
The contact layer method in the problem of bending of a multilayer beam. Fourier series solution
}

\author{
Andreev Vladimir I. ${ }^{1, \text { a }}$, Turusov Robert A. ${ }^{1, b}$, Tsybin Nikita Yu. ${ }^{1, c}$ \\ ${ }^{1}$ Moscow State University of Civil Engineering (National Research University), \\ 26 Yaroslavskoye Shosse, Moscow, 129337, Russia

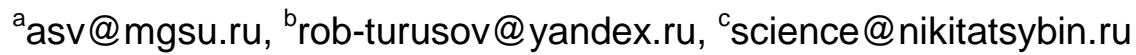

Keywords: Multilayer beam, Composite, Adhesive, Substrate, Bending, Contact layer, Adhesive interaction, Fourier series, Edge effect

\begin{abstract}
The article deals with the solution of the problem of bending of a hinged multilayer beam under the normal uniformly distributed load and induced axial forces. The interaction between layers is accomplished by the contact layer in which the substances of adhesive and substrate are mixed. We will consider the contact layer as the transversal anisotropic medium with such parameters that it can be represented as a set of short elastic rods, which are not connected to each other. The solution is obtained in the form of decomposition into Fourier series of sines. There is an example of the calculation of a three-layer beam. The convergence of the obtained solution is analyzed according to the number of accounted members of the decomposition
\end{abstract}

\section{Introduction}

The solution of the problem of the bending of a multilayer beam is of great complexity, since the order of the resolving system of equations is proportional to the number of layers. Analytical solutions can be obtained only for two and three-layer beams. For the solution, numerical methods are most often used. In this paper, the authors propose an analytic solution in the form of decomposition into Fourier series.

\section{Statement of Problem}

The Initial System of Equations. The initial system of differential equations obtained in [1] for a multilayer beam bending problem (Fig. 1) can be written as

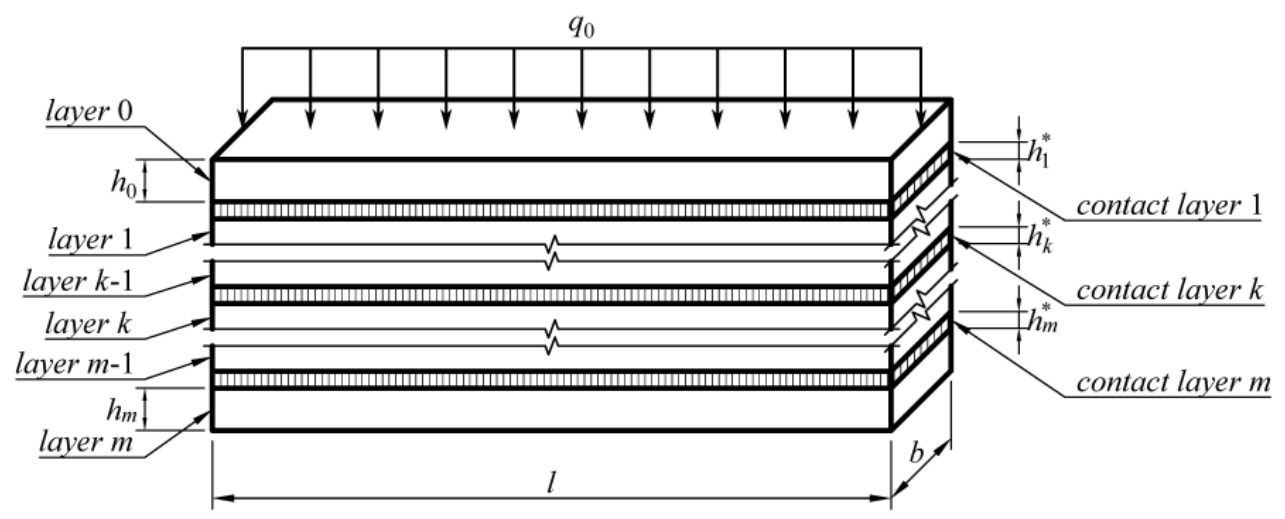

Fig. 1 Model of multilayer beam. 


$$
\left.\begin{array}{l}
D_{k} \cdot \frac{d^{4} v_{k}}{d x^{4}}=q_{k}+e_{k}^{-} \cdot b \cdot g_{k}^{*} \cdot\left(\frac{N_{k}+N_{f, k}}{B_{k}}-\frac{N_{k-1}+N_{f, k-1}}{B_{k-1}}+\frac{d^{2} v_{k}}{d x^{2}} \cdot e_{k}^{-}+\frac{d^{2} v_{k-1}}{d x^{2}} \cdot e_{k-1}^{+}\right)+ \\
+e_{k}^{+} \cdot b \cdot g_{k+1}^{*} \cdot\left(\frac{N_{k+1}+N_{f, k+1}}{B_{k+1}}-\frac{N_{k}+N_{f, k}}{B_{k}}+\frac{d^{2} v_{k+1}}{d x^{2}} \cdot e_{k+1}^{-}+\frac{d^{2} v_{k}}{d x^{2}} \cdot e_{k}^{+}\right)- \\
-e_{k}^{*} \cdot b \cdot\left(v_{k}-v_{k-1}\right)+e_{k+1}^{*} \cdot b \cdot\left(v_{k+1}-v_{k}\right) ; \\
\frac{d^{2} N_{k}}{d^{2} x}=g_{k}^{*} \cdot b \cdot\left(\frac{N_{k}+N_{f, k}}{B_{k}}-\frac{N_{k-1}+N_{f, k-1}}{B_{k-1}}+\frac{d^{2} v_{k}}{d x^{2}} \cdot e_{k}^{-}+\frac{d^{2} v_{k-1}}{d x^{2}} \cdot e_{k-1}^{+}\right)- \\
-g_{k+1}^{*} \cdot b \cdot\left(\frac{N_{k+1}+N_{f, k+1}}{B_{k+1}}-\frac{N_{k}+N_{f, k}}{B_{k}}+\frac{d^{2} v_{k+1}}{d x^{2}} \cdot e_{k+1}^{-}+\frac{d^{2} v_{k}}{d x^{2}} \cdot e_{k}^{+}\right) .
\end{array}\right\}
$$

The Eq. (1) are composed of the following values:

$v_{k}, N_{k}$-displacement and the axial force in the layer $k$ respectively;

$D_{k}, B_{k}$-averaged bending stiffness and tensile stiffness of the layer $k$ is determined from the expressions

$$
B_{k}=\int_{F_{k}} E_{k} d F_{k} ; D_{k}=\int_{F_{k}} E_{k} \cdot y_{k}^{2} d F_{k} ;
$$

$E_{k}$-Young's modulus of layer $k$;

$b$ - beam width;

$e_{k}^{-}, e_{k}^{+}-$the distance from the top and bottom edge of the layer $k$ to the neutral axis respectively,

$$
e_{k}^{-}=\frac{\int_{0}^{h_{k}} E_{k}\left(\xi_{k}\right) \cdot \xi_{k} d \xi_{k}}{\int_{0}^{h_{k}} E_{k}\left(\xi_{k}\right) d \xi_{k}} ; e_{k}^{+}=h_{k}-e_{k}^{-} ;
$$

$\xi_{k}$ - variable, calculated from the top edge of the layer $k$;

$h_{k}$ - thickness of layer $k$;

$g_{k}^{*}, e_{k}^{*}-$ averaged shear stiffness and tensile stiffness of the layer $k$ is determined from the expressions,

$$
g_{k}^{*}=\frac{G_{k}^{*}}{h_{k}^{*}} ; e_{k}^{*}=\frac{E_{k}^{*}}{h_{k}^{*}} \text {. }
$$

Here and further, all values marked with * refer to a contact layer.

$N_{f, k}$-induced forces in the layer $k$ due to the presence of pre-tension, temperature etc.;

$q_{k}$ - normal uniformly distributed load in the layer $k$.

From the system of equations (1) by substituting $k=0 \ldots m$, where $m+1$ is the number of layers, it is possible to obtain special cases of resolving equations.

\section{Boundary Conditions.}

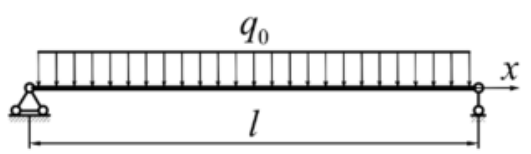

Fig. 2 The model.

We will consider the hinged beam (Fig. 2), the boundary conditions for which can be written as: 


$$
v_{k}(0)=v_{k}(l)=\frac{d^{2}}{d x^{2}}\left[v_{k}(0)\right]=\frac{d^{2}}{d x^{2}}\left[v_{k}(l)\right]=N_{k}(0)=N_{k}(l)=0 .
$$

For these boundary conditions, the solution of the system of equations (1) can be obtained in the form of decomposition into the Fourier series of sines, as in this case all the boundary conditions will be satisfied.

\section{The Decomposition of Functions in Fourier Series}

The Decomposition of Initial Functions in Fourier Series. In general case the decomposition of an arbitrary function into Fourier series of sines with a period of $T=l$ in the interval $x=0 \ldots l$ written as

$$
f(x)=\sum_{n=1}^{\infty} f_{n} \sin \left(\lambda_{n} x\right), \lambda_{n}=\frac{\pi n}{l} .
$$

Thus, we obtain

$$
\left.\begin{array}{l}
v_{k}=\sum_{n=1}^{\infty} v_{k}^{n} \sin \left(\lambda_{n} x\right) ; \frac{d^{2} v_{k}}{d x^{2}}=-\lambda_{n}^{2} \sum_{n=1}^{\infty} v_{k}^{n} \sin \left(\lambda_{n} x\right) ; \frac{d^{4} v_{k}}{d x^{4}}=\lambda_{n}^{4} \sum_{n=1}^{\infty} v_{k}^{n} \sin \left(\lambda_{n} x\right) ; \\
N_{k}=\sum_{n=1}^{\infty} N_{k}^{n} \sin \left(\lambda_{n} x\right) ; \frac{d^{2} N_{k}}{d x^{2}}=-\lambda_{n}^{2} \sum_{n=1}^{\infty} N_{k}^{n} \sin \left(\lambda_{n} x\right) .
\end{array}\right\}
$$

The normal uniformly distributed load and induced forces also need to be decomposed in Fourier series

$$
\left.\begin{array}{l}
q_{k}=\sum_{n=1}^{\infty} q_{k}^{n} \sin \left(\lambda_{n} x\right) ; \\
N_{f, k}=\sum_{n=1}^{\infty} N_{f, k}^{n} \sin \left(\lambda_{n} x\right),
\end{array}\right\}
$$

where

$$
\left.\begin{array}{l}
q_{k}^{n}=\frac{2 \cdot q_{k}}{l} \cdot \int_{0}^{l} \sin \left(\lambda_{n} x\right) d x=\frac{4 \cdot q_{k} \cdot \sin ^{2}\left(\frac{\pi \cdot n}{2}\right)}{\pi \cdot n} ; \\
N_{f, k}^{n}=\frac{2 \cdot N_{f, k}}{l} \cdot \int_{0}^{l} \sin \left(\lambda_{n} x\right) d x=\frac{4 \cdot N_{f, k} \cdot \sin ^{2}\left(\frac{\pi \cdot n}{2}\right)}{\pi \cdot n} .
\end{array}\right\}
$$

The Transformation of the Initial System of Equations. Let us substitute expressions (4) and (5) in the system of Eq. (1). After transformations we obtain a system of two resolving algebraic equations for the layer $k$.

$$
\left.\begin{array}{l}
\alpha_{k, k-1}^{n} \cdot v_{k-1}^{n}+\beta_{k, k-1} \cdot N_{k-1}^{n}+\alpha_{k, k}^{n} \cdot v_{k}^{n}+\beta_{k, k} \cdot N_{k}^{n}+\alpha_{k, k+1}^{n} \cdot v_{k+1}^{n}+\beta_{k, k+1} \cdot N_{k+1}^{n}=F_{k}^{n} ; \\
\xi_{k, k-1}^{n} \cdot v_{k-1}^{n}+\psi_{k, k-1} \cdot N_{k-1}^{n}+\xi_{k, k}^{n} \cdot v_{k}^{n}-\psi_{k, k}^{n} \cdot N_{k}^{n}+\xi_{k, k+1}^{n} \cdot v_{k+1}^{n}+\psi_{k, k+1} \cdot N_{k+1}^{n}=f_{k}^{n},
\end{array}\right\}
$$

in which 


$$
\left.\begin{array}{l}
\alpha_{k, k-1}^{n}=e_{k-1}^{+} \cdot e_{k}^{-} \cdot g_{k}^{*} \cdot \lambda_{n}^{2}-e_{k}^{*} ; \beta_{k, k-1}=\frac{e_{k}^{-} \cdot g_{k}^{*}}{B_{k-1}} ; \\
\alpha_{k, k}^{n}=\frac{D_{k}}{b} \cdot \lambda_{n}^{4}+\lambda_{n}^{2} \cdot\left[\left(e_{k}^{-}\right)^{2} \cdot g_{k}^{*}+\left(e_{k}^{+}\right)^{2} \cdot g_{k+1}^{*}\right]+e_{k}^{*}+e_{k+1}^{*} ; \beta_{k, k}=\frac{1}{B_{k}} \cdot\left(e_{k}^{+} \cdot g_{k+1}^{*}-e_{k}^{-} \cdot g_{k}^{*}\right) ; \\
\alpha_{k, k+1}^{n}=e_{k+1}^{-} \cdot e_{k}^{+} \cdot g_{k+1}^{*} \cdot \lambda_{n}^{2}-e_{k+1}^{*} ; \beta_{k, k+1}=-\frac{e_{k}^{+} \cdot g_{k+1}^{*}}{B_{k+1}} ; \\
F_{k}^{n}=\frac{q_{k}^{n}}{b}+e_{k}^{-} \cdot g_{k}^{*} \cdot\left(\frac{N_{f, k}^{n}}{B_{k}}-\frac{N_{f, k-1}^{n}}{B_{k-1}}\right)+e_{k}^{+} \cdot g_{k+1}^{*} \cdot\left(\frac{N_{f, k+1}^{n}}{B_{k+1}}-\frac{N_{f, k}^{n}}{B_{k}}\right) ; \\
\xi_{k, k-1}^{n}=g_{k}^{*} \cdot \lambda_{n}^{2} \cdot e_{k-1}^{+} ; \psi_{k, k-1}=\frac{g_{k}^{*}}{B_{k-1}} ; \xi_{k, k}^{n}=\lambda_{n}^{2} \cdot\left(g_{k}^{*} \cdot e_{k}^{-}-g_{k+1}^{*} \cdot e_{k}^{+}\right) ; \psi_{k, k}^{n}=-\frac{\lambda_{n}^{2}}{b}-\frac{1}{B_{k}} \cdot\left(g_{k}^{*}+g_{k+1}^{*}\right) ; \\
\xi_{k, k+1}^{n}=-g_{k+1}^{*} \cdot \lambda_{n}^{2} \cdot e_{k+1}^{-} ; \psi_{k, k+1}=\frac{g_{k+1}^{*}}{B_{k+1}} ; f_{k}^{n}=g_{k}^{*} \cdot\left(\frac{N_{f, k}^{n}}{B_{k}}-\frac{N_{f, k-1}^{n}}{B_{k-1}}\right)-g_{k+1}^{*} \cdot\left(\frac{N_{f, k+1}^{n}}{B_{k+1}}-\frac{N_{f, k}^{n}}{B_{k}}\right) .
\end{array}\right\}
$$

The system of resolving equations for the beam can be obtained from the equations (6) and represented in matrix form for the $n$-th member of the decomposition as follows:

$$
[A]_{n} \cdot[x]_{n}=[\eta]_{n},
$$

where

$[A]_{n}$ - coefficient matrix of size $2 \cdot m \times 2 \cdot m$;

$[x]_{n}$ - vector of unknowns of length $2 \cdot m$;

$[\eta]_{n}$ - vector of loads of length $2 \cdot m$.

The solution of the system of Eq. (8) is the expression

$$
[x]_{n}=[\eta]_{n} \cdot[A]_{n}^{-1} .
$$

Calculating the coefficients in the first and the last two rows of the matrix $[A]_{n}$ and the vector $[\eta]_{n}$ it is necessary to consider that $g_{0}^{*}=g_{m+1}^{*}=e_{0}^{*}=e_{m+1}^{*}=0$.

\section{Example of Calculation}

A Three-Layer Beam Model. Resolving Equations for the Three-Layer Beam. As an example of calculation, we will consider a three-layer beam (Fig. 3). This beam consists of two outer layers of carbon fiber and an inner layer of syntactic based on hollow glass microspheres. Similar materials are increasingly used in aircraft construction and mechanical engineering where it is necessary to ensure high stiffness, strength and lower mass.

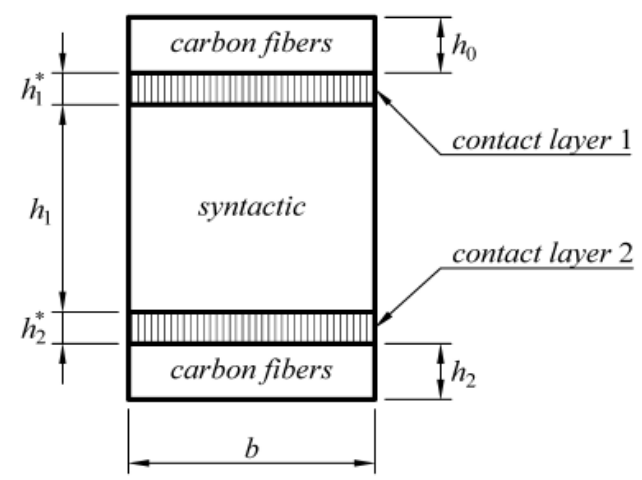

Fig. 3 Three-layer beam model. 
It should be noted that the layers of epoxy resin that bind the substrate are not taken into account in this scheme.

In this case, the resolving system of Eq. (8) takes the form

$$
\left[\begin{array}{cccccc}
\alpha_{0,0}^{n} & \beta_{0,0} & \alpha_{0,1}^{n} & \beta_{0,1} & 0 & 0 \\
\xi_{0,0}^{n} & \psi_{0,0}^{n} & \xi_{0,1}^{n} & \psi_{0,1} & 0 & 0 \\
\alpha_{1,0}^{n} & \beta_{1,0} & \alpha_{1,1}^{n} & \beta_{1,1} & \alpha_{1,2}^{n} & \beta_{1,2} \\
\xi_{1,0}^{n} & \psi_{1,0}^{n} & \xi_{1,1}^{n} & \psi_{1,1} & \xi_{1,2}^{n} & \psi_{1,2} \\
0 & 0 & \alpha_{2,1}^{n} & \beta_{2,1} & \alpha_{2,2}^{n} & \beta_{2,2} \\
0 & 0 & \xi_{2,1}^{n} & \psi_{2,1} & \xi_{2,2}^{n} & \psi_{2,2}
\end{array}\right] \cdot\left[\begin{array}{c}
v_{0}^{n} \\
N_{0}^{n} \\
v_{1}^{n} \\
N_{1}^{n} \\
v_{2}^{n} \\
N_{2}^{n}
\end{array}\right]=\left[\begin{array}{c}
F_{0}^{n} \\
f_{0}^{n} \\
F_{1}^{n} \\
f_{1}^{n} \\
F_{2}^{n} \\
f_{2}^{n}
\end{array}\right] .
$$

The expressions for the $n$-th members of the Fourier series are not listed in this article, as they are cumbersome.

The following geometrical and physical-mechanical characteristics [2] are applied by the calculation

$$
\begin{aligned}
& h_{0}=h_{2}=2 \mathrm{~mm} ; h_{1}=30 \mathrm{~mm} ; b=10 \mathrm{~mm} ; E_{0}=E_{2}=3.5 \cdot 10^{5} \mathrm{MPa} ; E_{1}=2.6 \cdot 10^{3} \mathrm{MPa} \\
& q=1 \frac{\mathrm{kN}}{\mathrm{m}} ; l=500 \mathrm{~mm} ; g_{1}^{*}=g_{2}^{*}=10^{4} \frac{\mathrm{MPa}}{\mathrm{mm}} ; e_{1}^{*}=e_{2}^{*}=10^{4} \frac{\mathrm{MPa}}{\mathrm{mm}} .
\end{aligned}
$$

From the solution of the system (10) displacement and axial forces are determined for each of the layers. The remaining parameters of the beam are expressed through them as follows

$$
\left.\begin{array}{l}
M_{k}=-D_{k} \cdot \frac{d^{2} v_{k}}{d x^{2}} ; Q_{k}=-D_{k} \cdot \frac{d^{3} v_{k}}{d x^{3}}+\tau_{k}^{*} \cdot b \cdot e_{k}^{-}+\tau_{k+1}^{*} \cdot b \cdot e_{k}^{+} ; \\
\varphi_{k}=\frac{d v_{k}}{d x} ; \tau_{1}^{*}=-\frac{1}{b} \cdot \frac{d N_{1}}{d x} ; \tau_{2}^{*}=\frac{1}{b} \cdot \frac{d N_{2}}{d x} .
\end{array}\right\}
$$

The Research of the Convergence of the Analytical Solutions in the Series in Comparison to the Numerical Solution. Below are the curves for the displacement in the middle of the beam and tangential stresses on the edge of the section. These curves demonstrate an error of the series solution compared to the numerical solution for various number of decomposition members. For the other parameters, the curves are similar.

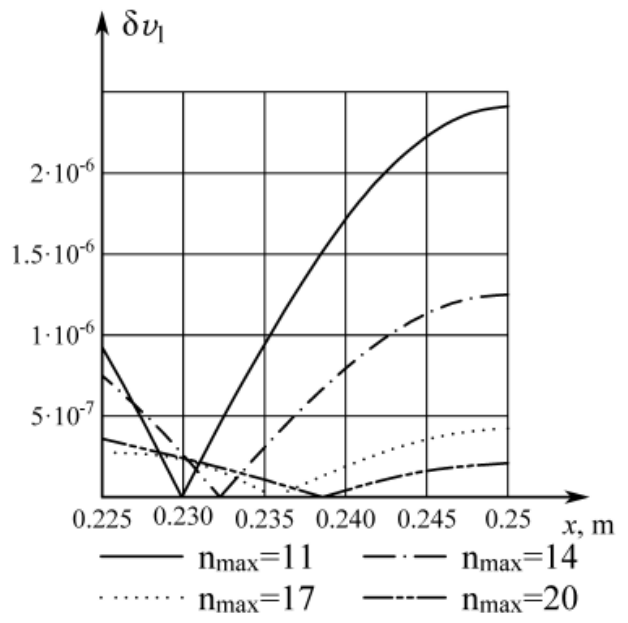

Fig. 4 Error of displacement at layer 1.

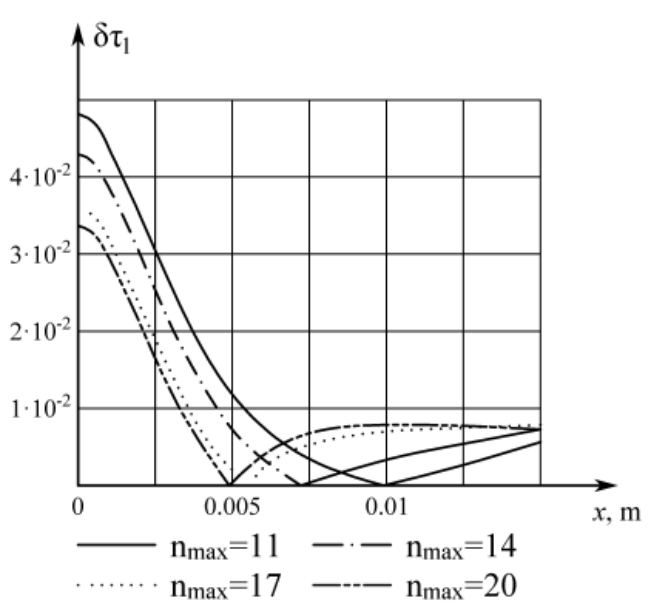

Fig. 5 Error of tangential stress of contact layer 1.

For the numerical solution of the initial system of equations Runge-Kutt method of 5-th order is applied with the maximum acceptable error of $10^{-8}$. 
The error is calculated as follows:

$$
\delta f(x)=\left|\frac{f_{\text {num. }}(x)}{f_{\text {ser. }}\left(x, n_{\max }\right)}-1\right|,
$$

where, $n_{\max }$ - number of accounted members of the decomposition.

The convergence of current values of layer 0 analyzed separately due to the presence of edge effects. Below this curves

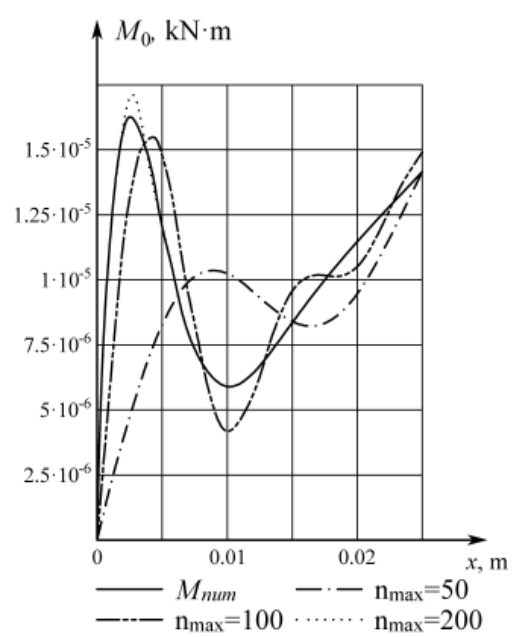

Fig. 6 Bending moment of layer 0 .

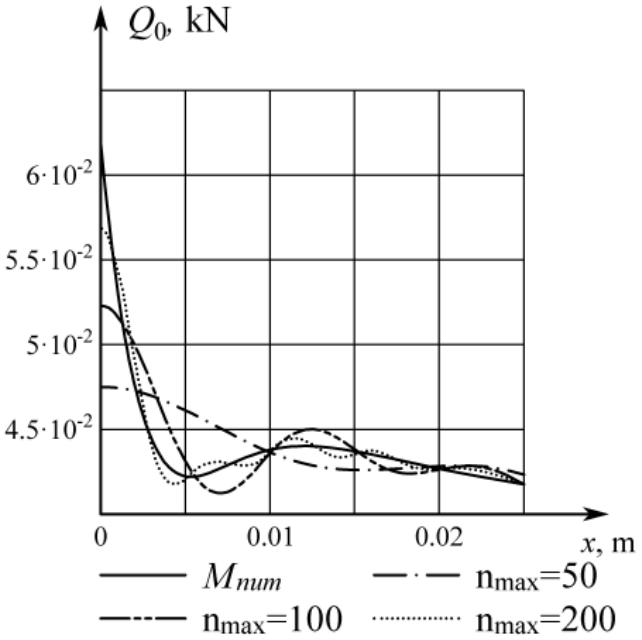

Fig. 7 Shear force of layer 0.

Below are relative curves that reflect the changes of the stress-strain state of a three-layer beam in layer 1 at 20 accounted decomposition members and in the layer 0 at 500 decomposition members.

The valuation is obtained according to the following expression:

$$
\bar{f}(x)=\frac{f(x)}{\max [f(x)]} \text {. }
$$

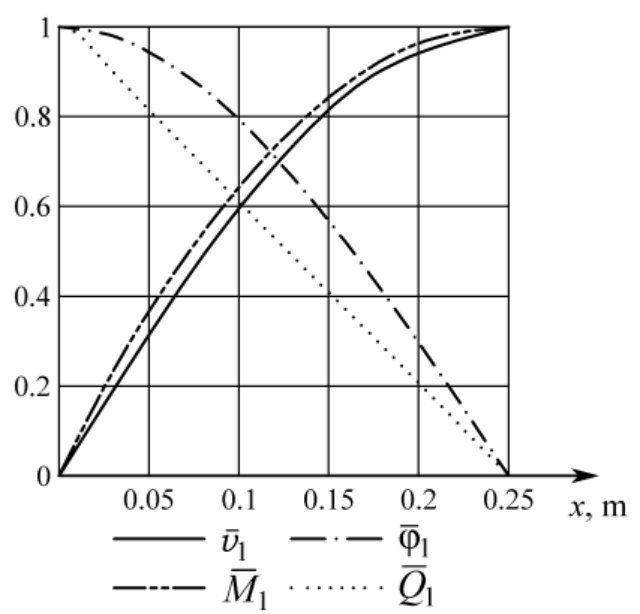

Fig. 8. Displacement, rotation angle, bending moment and shear force of layer 1 .

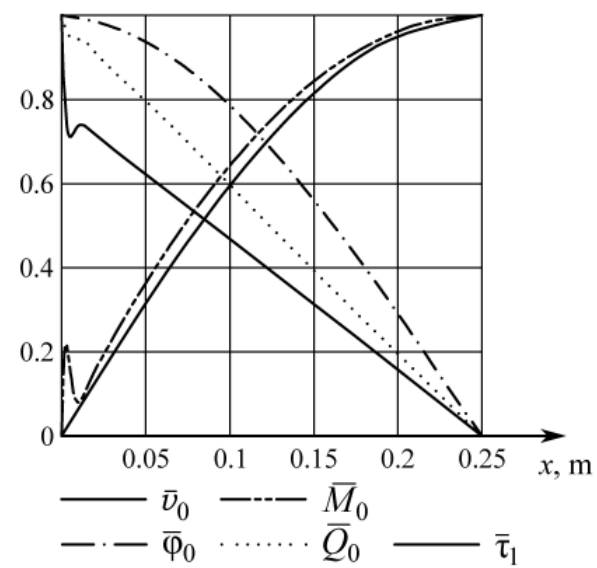

Fig. 9. Displacement, rotation angle, bending moment, shear force of layer 0 and tangential stress of contact layer 1 .

The axial forces are not displayed on the presented graphs due to the fact that the nature of their distribution coincides with the distribution of bending moments. 


\section{Conclusion}

The method of solving the problem of the bending of a hinged multilayer beam has been developed in the form of the decomposition in Fourier series of sines. This method is presented in a matrix form. As a result, the solution can be easily obtained with the use of software of linear algebra.

It should be noted that the series for displacements, rotation angles, bending moments and axial forces are fast enough to converge. Therefore, a solution with an acceptable error of $5 \%$ can be obtained at 10 accounted members of the decomposition. For the transversal forces and tangential stresses, the solution with 20 accounted decomposition members has a similar error. However, for the analysis of stress-strain state in the zone of the edge effect it is necessary to consider solutions based on circa 200-500 decomposition members.

Material of strength and creep of polymer materials can also be found in the works [3 -7].

\section{References}

[1] V. I. Andreev, R. A. Turusov, N. Yu. Tsybin, Application of the contact layer in the solution of the problem of bending the multilayer beam. Proc. Eng. 153 (2016) 59-65.

[2] A. M. Kuperman, R. A. Turusov, A. J. Gorenberg, V. I. Solodilov, R. A. Korokhin, Y. A. Gorbatkina, V. G. Ivanova-Mumzhieva, O. A. Zhuravleva, A. V. Baikov, Shear elastic and strength characteristics of syntactics based on hollow glass microspheres. Mech. Comp. Mater. 50(6) (2015) 705-716.

[3] N. Yu. Tsybin, R. A. Turusov, V. I. Andreev, Comparison of creep in free polymer rod and creep in polymer layer of the layered composite. Proc. Eng. 153 (2016) 51-58.

[4] V. I. Andreev, R. A. Turusov, N. Yu. Tsybin, Long strength of layered composite under normal fracture. Adv. Eng. Res. 39 (2015) 1703-1708.

[5] R. A. Turusov, A. M. Kuperman, V. I. Andreev, Determining the True Strength of the Material of Fiberglass Thick Rings When Stretched with Half-disks. Adv. Mater. Res. 1102 (2015) 155-159

[6] V. I. Andreev, B. M. Yazyev, A. S. Chepurnenko, On the bending of a thin polymer plate at nonlinear creep. Adv. Mater. Res. 900 (2014) 707-710.

[7] V. I. Andreev, A. S. Chepurnenko, B. M. Yazyev, Energy method in the calculation stability of compressed polymer rods considering creep. Adv. Mater. Res. 1004-1005 (2014) 257-260. 